Tropical Journal of Pharmaceutical Research October 2020; 19 (10): 2227-2233

ISSN: $1596-5996$ (print); 1596-9827 (electronic)

(C) Pharmacotherapy Group, Faculty of Pharmacy, University of Benin, Benin City, 300001 Nigeria.

Available online at http://www.tjpr.org

Original Research Article

http://dx.doi.org/10.4314/tjpr.v19i10.29

\title{
Therapeutic effect of teriparatide combined with low- frequency pulsed electromagnetic field on hip fracture
}

\author{
Xiang Sun, Xuefeng Guo, Wen Cao, Qiang Dong* \\ Department of Traumatology, Tianjin Hospital, Tianjin 300211, China \\ ${ }^{*}$ For correspondence: Email: qiangdong452@yandex.com
}

Sent for review: 11 June 2020

Revised accepted: 26 September 2020

\begin{abstract}
Purpose: To evaluate the therapeutic effect of teriparatide in combination with low-frequency pulsed electromagnetic field in the management of hip fracture.

Methods: Patients with hip fracture internal fixation and under conventional postoperative basic adjuvant therapy were compared with those patients who received teriparatide combined with low frequency pulsed electromagnetic field after operation. The content of Bone-Specific Alkaline Phosphatase (BALP), type I Procollagen Carboxy Terminal Propeptide (PICP), serum bone glaprotein $(B G P), \mathrm{Ca}^{2+}$, hip function and degree of bone scab formation were evaluated using Harris score and Fernadez-esteve scoring system. Kaplan-Meier survival curve was plotted to analyze differences in short-term prognosis of different postoperative adjuvant treatment measures.

Results: Harris score of the hip joint and the effect of bone formation in the experimental group were higher than that of the control group. The blood levels of BGP, BALP, PICP and serum $\mathrm{Ca}^{2+}$ in the experimental group were higher than that of the control group $(p<0.05)$. The plotted Kaplan-Meier survival curve indicates that the prognosis of the experimental group was better than that of control group $(p<0.05)$.

Conclusion: The adjuvant therapy of teriparatide combined with low-frequency pulsed electromagnetic field can improve the recovery function and enhance the prognosis of patients who underwent hip fracture surgery.
\end{abstract}

Keywords: Teriparatide; Low frequency pulsed electromagnetic field, hip fracture

\begin{abstract}
This is an Open Access article that uses a funding model which does not charge readers or their institutions for access and distributed under the terms of the Creative Commons Attribution License (http://creativecommons.org/licenses/by/4.0) and the Budapest Open Access Initiative (http://www.budapestopenaccessinitiative.org/read), which permit unrestricted use, distribution, and reproduction in any medium, provided the original work is properly credited.

Tropical Journal of Pharmaceutical Research is indexed by Science Citation Index (SciSearch), Scopus, International Pharmaceutical Abstract, Chemical Abstracts, Embase, Index Copernicus, EBSCO, African Index Medicus, JournalSeek, Journal Citation Reports/Science Edition, Directory of Open Access Journals (DOAJ), African Journal Online, Bioline International, Open-J-Gate and Pharmacy Abstracts
\end{abstract}

\section{INTRODUCTION}

Hip joint is the important joint in the body that supports the body weight when there is a movement. In case of an accident or a fall, hip joint is prone to different fractures including acetabular fractures and intertrochanteric fracture. Based on the magnitude of the fracture or impact, the movement of hip joint becomes limited and exhibits symptoms, for instance, pain [1-3]. The anatomical position of the hip joint is deep and internal fixation is generally used to treat it $[4,5]$. Despite the effective treatment of internal fixation, some patients still suffer from postoperative loosening and poor fracture healing $[2,6]$. So, anti-osteoporotic drugs can be used together with the usual regimen for patients who underwent hip joint surgery. This is to 
ensure that it further improves the effect of hip joint internal fixation surgery.

Tripathin is a synthetic parathyroid hormone analogue which is used by physicians for a long time in practical clinical applications to improve the symptoms of osteoporosis patients so as to mitigate the risks of fractures $[7,8]$. Recent studies concluded that the treatment with Teriparatide, after vertebroplasty, improved the effect of fracture healing and bone density in patients with osteoporosis [9]. Low-frequency pulsed electromagnetic field can accelerate the growth of osteoblasts while it can decelerate the maturing ability of osteoblasts through indirect effect in in vitro magnetic field. This, in turn, promotes the growth of callus at the fracture site and assists in its healing $[10,11]$. However, the studies focusing the adjuvant therapeutic effect of teriparatide combined with low-frequency pulsed electromagnetic field in patients with hip fractures were either negligible or not conducted at all. Therefore, in order to understand its therapeutic effect, the current study applied teriparatide, in combination with low-frequency pulsed electromagnetic field, during postoperative adjuvant treatment of hip fracture. Further, the study also evaluated the practical application through the data obtained from postoperative hip function observations, bone metabolism and biochemical indexes.

\section{METHODS}

\section{General clinical profile of patients}

A total of 148 patients, with hip fracture, treated in the Department of Orthopedics, Tianjin Hospital, Tianjin, was studied. The study period was from January 2018 to January 2019 and the patients had an average age of $54.63 \pm 10.86$ years. After the surgery, the patients were divided into control group $(\mathrm{n}=65)$ and experimental group $(n=83)$ following auxiliary fracture healing method. The experimental group was treated with routine postoperative treatment and additional auxiliary treatment i.e., teriparatide added in the treatment regimen in combination with low-frequency pulsed electromagnetic field. The inclusion criteria for the study were (1) Confirmed hip fracture patients as per practical osteology and established by X-ray and other imaging results and clinical symptoms [12]; (2) Those patients who underwent internal fixation in the study hospital within $24 \mathrm{~h}$ after hip fracture and (3) patients without any diagnosed mental disorder were included in the study. The exclusion criteria for the study were (1) long-term usage of hormones for treatment due to other diseases; (2) patients suffering from severe endocrine system disorders such as T1D, T2D, Thyroid, Osteoporosis etc at the time of treatment and (3) patients with hypersensitivity towards teriparatide. Ethical approval for the study was given by the institutional ethical review board (approval no. Qiang/2019/01) of Tianjin Hospital, Tianjin 300211 , China. All the patients and their families were informed about the study purpose and obtained the informed consent. All the procedures, performed in this study, were in accordance with Helsinki declaration (1964) and its comparable ethical standards [13].

\section{Postoperative adjuvant therapy}

The control group received conventional postoperative auxiliary treatment measures as mentioned below. After internal fixation, the control group patients received antiinflammatory, detumescence treatment and subcutaneous injection of Wanmaishu (National drug approval board H20063910, Beishanshan Biochemical Pharmaceutical co., LTD.). This is to prevent the formation of deep venous thrombosis in lower limbs caused by limited physical movements in bed during post-surgery period. At the time of recovery, calcium and vitamin D were given orally to promote the healing of fracture and restorative exercise was started immediately after 2-3 days of surgery. Based on the progress observed in fracture healing during the post-treatment processes, the patient was encouraged to walk by themselves and lift heavy weights on a case-by-case condition.

In the experimental group, the following regimen was followed in parallel to the control group measures. Alike conventional anti-inflammatory and anti-dvt treatment, the patients were supplemented with calcium and vitamin $D$ to promote healing at fracture site. In addition to these, $20 \mathrm{~g}$ of teriparatide (S20150025, Lilly) was administered subcutaneously every day for three months. An electromagnetic field therapeutic instrument (BG100S, Beijing huayang limin) was used for auxiliary treatment at the fracture site. The parameters followed for the treatment were magnetic field intensity of 20 - 30gs, pulse frequency between 9-12 Hz, and the average treatment time was about 45 mins. In the $1^{\text {st }}$ and $2^{\text {nd }}$ month after the operation, the patients were treated once a day. During $3^{\text {rd }}$ and $4^{\text {th }}$ month, the patients were treated once in two days and during $5^{\text {th }}$ and $6^{\text {th }}$ month, the frequency changed to once in three days.

\section{Determination of surgical efficacy indices}

The Harris score of joint function was obtained before treatment and three months after the 
surgery. The score is inclusive of the following aspects: (1) pain, (2) performing day-to-day activities and (3) the range of motion of the joint. The total score is 100 points and the score is directly proportional to the degree of recovery of hip joint function.

Fasting blood was collected from the patients before treatment and three months after surgery. The serum calcium level in the blood of both the groups was analyzed using automatic blood biochemical analyzer (7870, Hitachi). ELISA kit (ym-s0838, Shanghai yuanmu; Ym-s09658, Shanghai yuanmu) was used to detect the gamma-Carboxyglutamic acid-containing protein of bone (BGP), procollagen I carboxyterminal propeptide (PICP) and serum bone alkaline phosphatase (BALP) in serum.

X-rays were taken after three months of surgery. Fernadez - esteve rating score was used to study the impact of callus formation. The scores and the relevant interpretation are as follows; I : no radiology callus formation. $\Pi$ : cloudy callus near fracture end; III: X-ray indicates the callus formation on the right side of the fracture; IV: formation of callus is openly visible in X-ray image on both sides of the fracture; $\mathrm{V}$ : an obvious structural callus.

\section{Postoperative follow-up}

The patients were followed up for one year after the operation during when they attended outpatient clinic for follow-up assessment. In this study, endpoint events were defined. The adverse prognosis was identified during follow-up which included: fracture nonunion, loosely-bound internal fixation, hip varus, reoccurrence of fractures and other complications that seriously affect the hip joint function. Deletion was defined as the loss of follow-up or death of the patient under study.

\section{Statistical analysis}

The rate of adoption for the experiment was expressed in $(\%)$ while the measurement data conforming to the normal distribution was expressed as mean \pm standard deviation. Two independent sample t-tests and chi-square tests were used to compare the differences in clinical data and Harris score between control and experimental groups. Wilcoxon rank-sum test was used to compare the difference in Fernadezesteve rating between the groups. The t-test of the two independent samples was also used to compare the differences between the groups in serum calcium ion water equality serum index. Finally, Kaplan-Meier survival curve was plotted to analyze the differences in the prognosis of different postoperative adjuvant treatment measures, and log-rank analysis was conducted. All the statistical analyses were conducted using Prism, and $P<0.05$ was considered to be statistically significant.

\section{RESULTS}

\section{General clinical profile of the patients}

The general clinical profile of the two study groups are shown in Table 1, and the results showed that there was no significant statistical difference in the general clinical data of the two groups $(p<0.05)$.

\section{Hip joint function and callus formation after surgery}

Harris scores of the patients for both the groups were recorded as shown in Table 2.

Table 1: General clinical profile of the patients

\begin{tabular}{|c|c|c|c|c|}
\hline Group & $\begin{array}{c}\text { Control } \\
\text { Group }(n=65)\end{array}$ & $\begin{array}{c}\text { Study } \\
\text { Group }(n=83)\end{array}$ & Statistics & $P$-value \\
\hline 1. Gender (male/female) & $45 / 20$ & $62 / 22$ & 0.382 & 0.538 \\
\hline 2. Age & $56.83 \pm 10.64$ & $53.61 \pm 12.09$ & 1.746 & 0.083 \\
\hline 3. $\mathrm{BMI}$ index $\left(\mathrm{kg} / \mathrm{m}^{2}\right)$ & $23.63 \pm 4.08$ & $24.01 \pm 3.18$ & -0.636 & 0.525 \\
\hline 4. Cause of fracture (case) & & & & \\
\hline a. The traffic accident & $24(36.92 \%)$ & $32(38.55 \%)$ & 0.271 & 0.847 \\
\hline b. Falling from high & $5(7.69 \%)$ & $8(9.64 \%)$ & & \\
\hline c. Falling & $36(55.39 \%)$ & $43(51.81 \%)$ & & \\
\hline 5. Fracture type (case) & & & & \\
\hline a. Femoral neck fracture & $34(52.31 \%)$ & $44(53.01 \%)$ & 0.046 & 0.977 \\
\hline b. Intertrochanteric fracture & $12(18.46 \%)$ & $16(19.28 \%)$ & & \\
\hline $\begin{array}{l}\text { c. Bottom of femoral neck to lower } \\
\text { trochanter }\end{array}$ & $19(29.23 \%)$ & $23(27.71 \%)$ & & \\
\hline
\end{tabular}


There was no significant statistical difference found in the preoperative Harris score between the groups $(p>0.05)$. However, the experimental group yielded significantly higher values than the control group at the $3^{\text {rd }}$ month of treatment $(p<$ $0.05)$. The accumulation of callus formation in both the groups is shown in Figure 1. Wilcoxon rank-sum test showed that the callus formation in the fracture site, of the experimental group, was superior to that of the control group $(p<0.05)$.

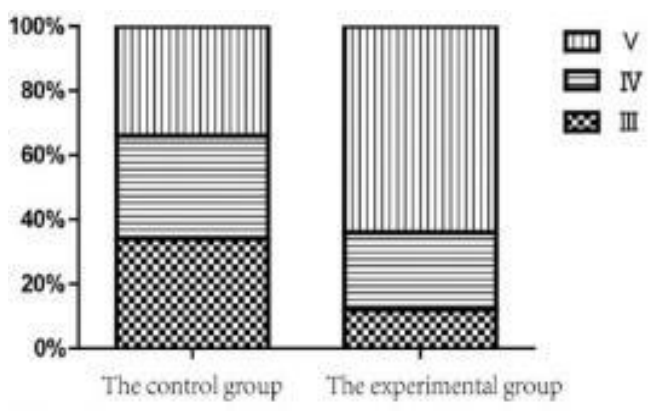

Figure 1: Ratings of Fernade-esteve

\section{Serum biochemical indices}

Serum biochemical indices for both the groups related to bone growth are shown in Table 3 . Three months after treatment, the blood levels of BGP, BALP and PICP in both control group and the experimental group were found to be higher than the corresponding serum factor levels before treatment. The three serological indices in the experimental group were found to be higher than the control group $(p<0.05)$. Figure 2 shows the blood $\mathrm{Ca}^{2+}$ concentration in both the groups. After $2^{\text {nd }}$ and $3^{\text {rd }}$ month of treatment, the blood $\mathrm{Ca}^{2+}$ concentration in the experimental group was found to be higher than that of the control group $(p<0.05)$.

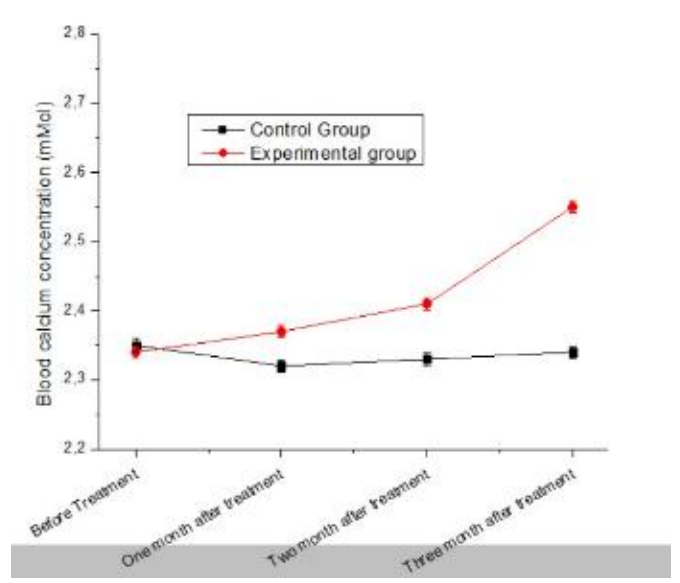

Figure 2: Variation curves of blood $\mathrm{Ca}^{2-}$ Concentration in the two group $\left({ }^{* *} p<0.001\right.$ compared with control group, ${ }^{* * *} p<0.001$, ns- statistically not significant)

\section{Survival curve analysis}

Figure 3 shows the postoperative situation of two groups of patients. The researchers ensured that there was no loss in the postoperative follow-up of the patients.

Table 2: Comparison of Harris scores

\begin{tabular}{lcccc}
\hline Group & Before study & After 3 months & T & $P$-value \\
\hline Observation $(n=65)$ & $54.08 \pm 6.83$ & $82.63 \pm 11.23$ & 13.99 & 0.00 \\
Control $(\mathrm{n}=83)$ & $56.83 \pm 10.64$ & $74.09 \pm 7.43$ & 10.75 & 0.00 \\
$\mathrm{~T}$ & -1.811 & 5.55 & & \\
$P$-value & 0.072 & 0.00 & & \\
\hline
\end{tabular}

Table 3: Bone biochemical related serum levels in the patients

\begin{tabular}{|c|c|c|c|c|c|}
\hline Variable & & Before & After 3 months & $t$ & $P$-value \\
\hline \multirow{2}{*}{$\mathrm{BGP}(\mu \mathrm{g} / \mathrm{L})$} & Study $(n=65)$ & $8.13 \pm 0.83$ & $32.63 \pm 5.54$ & -35.121 & 0.000 \\
\hline & \multirow[t]{3}{*}{ Control $(n=83)$} & $7.91 \pm 0.76$ & $24.08 \pm 7.43$ & -17.454 & 0.000 \\
\hline $\mathrm{T}$ & & 1.678 & 7.751 & & \\
\hline$P$-value & & 0.095 & 0.000 & & \\
\hline \multirow{2}{*}{ BALP(U/L) } & \multirow{2}{*}{$\begin{array}{l}\text { Observation } \\
\quad(n=65) \\
\text { Control }(n=83)\end{array}$} & $75.13 \pm 8.27$ & $165.63 \pm 21.09$ & -38.063 & 0.000 \\
\hline & & $73.62 \pm 9.42$ & $144.35 \pm 18.07$ & -27.066 & 0.000 \\
\hline \multirow{2}{*}{$\begin{array}{l}\mathrm{T} \\
P \text {-value }\end{array}$} & & 1.689 & 6.689 & & \\
\hline & & 0.093 & 0.000 & & \\
\hline \multirow{2}{*}{$\mathrm{PICP}(\mu \mathrm{g} / \mathrm{L})$} & $(n=65)$ & $135.98 \pm 20.14$ & $215.63 \pm 16.65$ & -24.298 & 0.000 \\
\hline & $(n=83)$ & $139.08 \pm 19.08$ & $184.53 \pm 23.06$ & -12.239 & 0.000 \\
\hline $\mathrm{T}$ & & -0.957 & 9.163 & & \\
\hline$P$-value & & 0.34 & 0.000 & & \\
\hline
\end{tabular}


In the experimental group, the number of patients with internal fixation device loosening were 4 while with recurrent fractures, it was 3 . The number of patients in the control group was 6 and 7 for the respective follow-ups i.e., internal fixation device loosening and recurrent fractures, respectively. The log-rank test results indicated that the surgical prognosis of the experimental group was better compared to control group ( $p<$ 0.05).

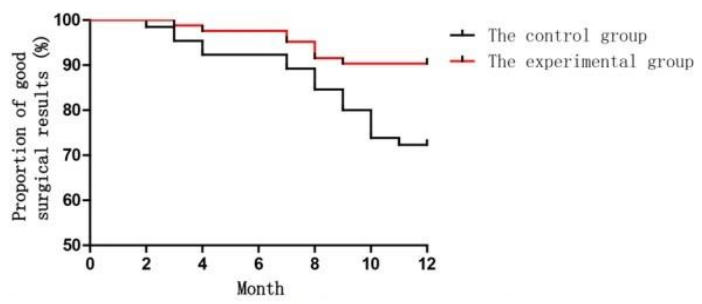

Figure 3: Effect of the surgical treatment on fracture

\section{DISCUSSION}

Osteoporosis and aging are high risk factors for hip fracture. So there is a wide range of difference observed in the proportion of hip fracture among different countries [14,15]. Although internal fixation treatment for fractures yielded satisfactory results in the past, some patients faced loose internal fixation devices, recurrent fractures and other phenomena, resulting in poor postoperative results. In the study, the authors administered teriparatide with a combination of low-frequency pulsed electromagnetic field treatment to heal the hip fracture after surgery. According to the results, it was found that teriparatide improved the therapeutic effect of hip joint surgery compared to the traditional method of supplementing calcium and vitamins after surgery.

Human parathyroid hormone is an important hormone secreted by parathyroid cells. This hormone can increase the concentration of $\mathrm{Ca}^{2+}$ in the blood by reabsorbing it in kidney and intestine. It also accelerates the excretion of phosphate through kidney, thereby increasing the density of the bone tissue in human body and promoting the healing at the fracture site $[16,17]$ Tripathin is a synthetic recombinant human parathyroid hormone analogue which can enact the role of human parathyroid hormone when subcutaneously injected in patients with fractures. It can stimulate postoperative healing in patients with fractures [18]. In previous studies, Sugie et al found that teriparatide was superior to other drugs such as zoledronic acid in postoperative adjuvant treatment of fracture patients. Further, it was also found that it significantly improved the bone mineral density in patients [20]. When thoracolumbar fracture patients were treated with teriparatide, a similar therapeutic effect was achieved $[9,12]$.

In this study, $20 \mathrm{~g}$ of teriparatide was administered subcutaneously every day for three months after internal fixation of hip fracture. When compared with conventional treatment, teriparatide significantly increased the concentration of $\mathrm{Ca}^{2+}$ among patients in the experimental group. This further improved the Harris score and callus forming ability of patients after surgery, which showed good application value. Since there may exist a certain proportion of osteoporotic patients among the older subjects in the study, the use of teriparatide after surgery tend to promote the healing of fracture ends while at the same time, it can also mitigate the symptoms of osteoporosis by increasing the concentration of $\mathrm{Ca}^{2+}$. However, it should be noted that the presence of excessive $\mathrm{Ca}^{2+}$ in the body may also leads to side effects, for instance, fatigue. So, prior to treatment regimen, both dosage and time should be checked. Further the blood $\mathrm{Ca}^{2+}$ concentration of patients should be dynamically monitored whenever necessary so as to obtain the best therapeutic effect.

One of the components of physical auxiliary treatment method is low frequency-pulsed electromagnetic field. The frequency as well as the specific intensity of the electromagnetic field are able to provide therapeutic effect directly on the osteoblasts of the fracture site. Both BGP [12] as well as BALP $[13,14]$ are secreted by mature osteoblasts. PICP is an important collagen in bone tissue which can reflect the activity of osteoblasts. These serum indicators are related to osteoblasts $[15,16]$. Few studies inferred that low-frequency electromagnetic fields tend to inhibit the growth of osteoclasts in fracture sites, reduce their bone-dissolving function and locally accelerate the blood flow to fracture site. These effects reduce the symptoms such as edema and hyperemia after fracture and significantly improve the prognosis $[10,11,18]$. In this study, it was found that when low-frequency pulsed electromagnetic field was applied, the levels of BGP, BALP and PICP in patients undergoing hip joint surgery were higher than that of the control group. This phenomenon infers that the magnetic field-assisted treatment accelerates the maturation of osteoblasts and promotes their osteogenic function.

\section{Limitations of the study}

Some of the limitations of the study are listed herewith. First, only a few studies conducted earlier followed the same methodology alike the 
current study. Otherwise, the sample size of the studies referred for this paper was small yet contained different criteria for groups. The current study combined these groups together because the effect of teriparatide was studied in a broad sense and not compared with a particular medication. Finally, though we adopted the proven methods to analyze the comparative data, a detailed and comprehensive analysis was restricted.

\section{CONCLUSION}

Teriparatide, when combined with low-frequency pulsed electromagnetic field, improves hip function and prognosis after hip fracture. Kaplanmeier survival curve further confirmed that the adjuvant therapy of teriparatide in combination with low-frequency pulsed electromagnetic field can reduce risks such as recurrence of fracture and loosening of internal fixation among postoperative patients.

\section{DECLARATIONS}

\section{Conflict of interest}

No conflict of interest is associated with this work.

\section{Contribution of authors}

We declare that this work was done by the authors named in this article and all liabilities pertaining to claims relating to the content of this article will be borne by the authors. Xiang Sun \& Qiang Dong - Literature search, Data analysis, Statistical analysis, Manuscript preparation, Manuscript review, Guarantor. Xuefeng Guo, Wen Cao \& Qiang Dong - Concepts, Design, Definition of intellectual content, Literature search, Experimental studies, Data acquisition, Manuscript editing

\section{Open Access}

This is an Open Access article that uses a funding model which does not charge readers or their institutions for access and distributed under the terms of the Creative Commons Attribution License (http://creativecommons.org/licenses/by/ 4.0) and the Budapest Open Access Initiative (http://www.budapestopenaccessinitiative.org/rea d), which permit unrestricted use, distribution, and reproduction in any medium, provided the original work is properly credited.

\section{REFERENCES}

1. Hönle W, Scheller A, Lerzer S, Schuh A. Manuelle Diagnostik am Hüftgelenk Clinical examination of the hip joint. MMW Fortschr Med. 2018; 160(17):56-61.

2. Peeters CM, Visser E, Van de Ree CL, Gosens T, Den Oudsten BL, De Vries J. Quality of life after hip fracture in the elderly: $A$ systematic literature review. Injury. 2016; 47(7):1369-1382.

3. Ferguson RJ, Palmer AJ, Taylor A, Porter ML, Malchau H, Glyn-Jones S. Hip replacement. Lancet. 2018; 392(10158):1662-1671.

4. Tagliafico A, Bignotti B, Rossi F, Sconfienza LM, Messina C, Martinoli C. Ultrasound of the Hip Joint, Soft Tissues, and Nerves. Semin Musculoskelet Radiol. 2017; 21(5):582-588.

5. Nogués $X$, Martinez-Laguna D. Update on osteoporosis treatment. Tratamiento de la osteoporosis. Med Clin (Barc). 2018; 150(12):479-486.

6. Sun Yingfei, Chu Kaiwei, Li Zhongshi. Effect of teriparatide on postoperative osteoporotic thoracolumbar vertebral compression fractures and its effect on bone density [J]. Journal of Clinical Military Medicine. 2019; 47(04):82-83.

7. Bilgin HM, Çelik F, Gem M, Akpolat V, Yıldız I, Ekinci A, Özerdem MS, Tunik S. Effects of local vibration and pulsed electromagnetic field on bone fracture: $A$ comparative study. Bioelectromagnetics. 2017; 38(5):339-348.

8. World Medical Association. Ethical principles for medical research involving human subjects. European journal of emergency medicine: official journal of the European Society for Emergency Medicine. 2001; 8(3):221.

9. Sánchez A, Masoni A, Morosano M, Vitali L, Levit $G$. Hiponatremia como factor de riesgo de fractura de cadera en sujetos añosos [Hyponatremia as a risk factor for hip fracture in elderly subjects]. Medicina (B Aires). 2017; 77(4):348.

10. Leder BZ. Parathyroid Hormone and Parathyroid Hormone-Related Protein Analogs in Osteoporosis Therapy. Curr Osteoporos Rep. 2017; 15(2):110-119.

11. Goltzman D. 2018. Physiology of Parathyroid Hormone. Endocrinol Metab Clin North Am. 47(4):743-758.

12. Righetti $M$, Wach $J$, Desmarchelier $R$, Coury $F$. Teriparatide treatment in an adult patient with hypophosphatasia exposed to bisphosphonate and revealed by bilateral atypical fractures. Joint Bone Spine. 2018; 85(3):365-367.

13. Collinge C, Favela J. Use of teriparatide in osteoporotic fracture patients. Injury. 2016; 247 (Suppl 1): S36-S38.

14. Guerriero F, Ricevuti G. Extremely low frequency electromagnetic fields stimulation modulates autoimmunity and immune responses: a possible immuno-modulatory therapeutic effect in neurodegenerative diseases. Neural Regen Res. 2016; 11(12):1888-1895.

15. Yessayan L, Moore C, Lu M, Yee J. Bone-specific alkaline phosphatase and bone turnover in African

Trop J Pharm Res, October 2020; 19(10): 2232 
American hemodialysis patients. Hemodial Int. 2017; 21(1):90-96.

16. Hegde SS, Revankar AV, Patil AK. Identification of bonespecific alkaline phosphatase in saliva and its correlation with skeletal age. Indian J Dent Res. 2018; 29(6):721-725.

17. Güvenç TS. Fragmented QRS and serum propeptide of type I procollagen in hypertensive patients: Putting another brick in the wall. Turk Kardiyol Dern Ars. 2016; 44(7):545-547.

18. Wu $S$, $Y u Q$, Sun $Y$, Tian J. Synergistic effect of a LPEMF and SPIONs on BMMSC proliferation, directional migration, and osteoblastogenesis. Am J Transl Res. 2018; 10(5):1431-1443. 